\title{
A Five-Dimensional Truncation of the Plane Incompressible Navier-Stokes Equations
}

\author{
Carlo Boldrighini ${ }^{\star 1}$ and Valter Franceschini ${ }^{\star 2}$ \\ ${ }^{1}$ Istituto Matematico, Università di Camerino, I-62032 Camerino, Italy \\ 2 Istituto Matematico, Università di Modena, I-41100 Modena, Italy
}

\begin{abstract}
A five-modes truncation of the Navier-Stokes equations for a two dimensional incompressible fluid on a torus is considered. A computer analysis shows that for a certain range of the Reynolds number the system exhibits a stochastic behaviour, approached through an involved sequence of bifurcations.
\end{abstract}

\section{Introduction}

In recent years much attention has been devoted to the study of simple differential or difference equations which, although deterministic, exhibit a transition, as some parameters go through certain values, to a stochastic behaviour. The equations which are studied often arise in a natural way as simplified models in fluid dynamics and in ecology. The best known examples are perhaps the models of Lorenz [1] and Henon [2]. In these models stochastic behaviour arises as a consequence of the appearance of an attractor of complicated structure which is called "strange attractor". Trajectories in a neighbourhood of the attractor appear to move in a completely erratic way. Phenomena of this kind are predicted, under certain hypothesis, by the mathematical theory of turbulence of Ruelle and Takens $[3,4]$.

In the following we consider a model obtained by a suitable five-modes truncation of the Navier-Stokes equations for a 2-dimensional incompressible fluid on a torus. We argue on the basis of computer analysis that in a certain range of the Reynolds number our system behaves in a way similar to that predicted by Ruelle and Takens. However the sequence of bifurcations through which the system goes over stochastic behaviour is quite peculiar, and different both from that considered in the theory of Ruelle and Takens, and from that of the other known models.

* Partially supported by G.N.F.M., C.N.R. 


\section{The Model}

Consider the equations

$$
\left\{\begin{array}{l}
\partial \mathbf{u} / \partial t+(\mathbf{u} \cdot \nabla) \mathbf{u}=-\nabla p+\mathbf{f}+v \Delta \mathbf{u} \\
\operatorname{div} \mathbf{u}=0 \\
\int_{T^{2}} \mathbf{u} d \mathbf{x}=\mathbf{0}
\end{array}\right.
$$

where $\mathbf{u}$ is the velocity field on the torus $T^{2}=[0,2 \pi] \times[0,2 \pi], p$ is the pressure, and $\mathbf{f}$ is a (periodic) volume force.

We expand $\mathbf{u}$ in Fourier series

$$
\mathbf{u}(\mathbf{x})=\sum_{\mathbf{k} \neq 0} e^{i \mathbf{k} \cdot \mathbf{x}} \gamma_{\mathbf{k}} \frac{\mathbf{k}^{\perp}}{|\mathbf{k}|}
$$

where $\mathbf{k}=\left(k_{1}, k_{2}\right)$ is a "wave vector" with integer components, $\mathbf{k}^{\perp}=\left(k_{2},-k_{1}\right)$, and the condition $\gamma_{\mathbf{k}}=-\bar{\gamma}_{-\mathbf{k}}$ (reality condition) holds. By expanding $p$ and $\mathbf{f}$ in similar series we get formally the following equations for $\left\{\gamma_{\mathbf{k}}\right\}_{\mathbf{k} \neq 0}$ :

$$
\dot{\gamma}_{\mathbf{k}}=-i \sum_{\mathbf{k}_{1}+\mathbf{k}_{2}+\mathbf{k}=0} \frac{\left(\mathbf{k}_{1}^{\perp} \cdot \mathbf{k}_{2}\right)\left(k_{2}^{2}-k_{1}^{2}\right)}{2\left|\mathbf{k}_{1}\right|\left|\mathbf{k}_{2}\right||\mathbf{k}|} \bar{\gamma}_{\mathbf{k}_{1}} \bar{\gamma}_{\mathbf{k}_{2}}-v|\mathbf{k}|^{2} \gamma_{\mathbf{k}}+f_{\mathbf{k}}
$$

Once $\left\{\gamma_{\mathbf{k}}\right\}_{\mathbf{k} \neq 0}$ is known, $p_{\mathbf{k}}$ is given by

$$
p_{\mathbf{k}}=-i \sum_{\mathbf{k}_{1}+\mathbf{k}_{2}+\mathbf{k}=0} \frac{\left(\mathbf{k}_{2} \cdot \mathbf{k}_{1}^{\perp}\right)\left(\mathbf{k}_{1} \cdot \mathbf{k}_{2}^{\perp}\right)}{\left|\mathbf{k}_{1}\right|\left|\mathbf{k}_{2}\right||\mathbf{k}|^{2}} \bar{\gamma}_{\mathbf{k}_{1}} \bar{\gamma}_{\mathbf{k}_{2}}+\tilde{f}_{\mathbf{k}}
$$

In (2.1), (2.2) $f_{\mathbf{k}}, \tilde{f}_{\mathbf{k}}$ are the components of $\mathbf{f}$ with respect to $\frac{\mathbf{k}^{\perp}}{|\mathbf{k}|} e^{i \mathbf{k} \cdot \mathbf{x}}$ and $\frac{\mathbf{k}}{|\mathbf{k}|} e^{i \mathbf{k} \cdot \mathbf{x}}$ respectively.

If $L$ is a set of wave vectors such that if $\mathbf{k} \in L$, also $-\mathbf{k} \in L$, we define the truncated Navier-Stokes equations as

$$
\begin{aligned}
& \dot{\gamma}_{\mathbf{k}}=-i \sum_{\substack{\mathbf{k}_{1}+\mathbf{k}_{2}+\mathbf{k}=0 \\
\mathbf{k}_{1}, \mathbf{k}_{2} \in L}} \frac{\left(\mathbf{k}_{1}^{\perp} \cdot \mathbf{k}_{2}\right)\left(k_{2}^{2}-k_{1}^{2}\right)}{2\left|\mathbf{k}_{1}\right|\left|\mathbf{k}_{2}\right||\mathbf{k}|} \bar{\gamma}_{\mathbf{k}_{1}} \bar{\gamma}_{\mathbf{k}_{2}}-v|\mathbf{k}|^{2} \gamma_{\mathbf{k}}+f_{\mathbf{k}}, \quad(\mathbf{k} \in L) \\
& \gamma_{\mathbf{k}}=-\bar{\gamma}_{-\mathbf{k}} .
\end{aligned}
$$

In this paper we take $L$ as the set of vectors $\mathbf{k}_{1}=(1,1), \mathbf{k}_{2}=(3,0), \mathbf{k}_{3}=(2,-1)$, $\mathbf{k}_{4}=(1,2), \mathbf{k}_{5}=(0,1)$ and their opposites. Equations $(2.3)$ with this choice of $L$ admit solutions in which some components are real and others pure imaginary. We shall consider one such solution, namely one in which

$$
\gamma_{\mathbf{k}_{1}}=\gamma_{1}, \quad \gamma_{\mathbf{k}_{2}}=-i \gamma_{2}, \quad \gamma_{\mathbf{k}_{3}}=\gamma_{3}, \quad \gamma_{\mathbf{k}_{4}}=i \gamma_{4}, \quad \gamma_{\mathbf{k}_{5}}=\gamma_{5}
$$

with real $\gamma_{i}$ 's. 
The equations become, after having changed time and length scales in order to have $v=1$ and to get rid of some factors, and taking a force acting on the mode $\mathbf{k}_{3}$ :

$$
\begin{aligned}
& \dot{\gamma}_{1}=-2 \gamma_{1}+4 \gamma_{2} \gamma_{3}+4 \gamma_{4} \gamma_{5} \\
& \dot{\gamma}_{2}=-9 \gamma_{2}+3 \gamma_{1} \gamma_{3} \\
& \dot{\gamma}_{3}=-5 \gamma_{3}-7 \gamma_{1} \gamma_{2}+r \\
& \dot{\gamma}_{4}=-5 \gamma_{4}-\gamma_{1} \gamma_{5} \\
& \dot{\gamma}_{5}=-\gamma_{5}-3 \gamma_{1} \gamma_{4} .
\end{aligned}
$$

We consider such equations because they are the simplest non trivial truncation with more than three modes. Three mode truncations are not considered because they seem to lead to non interesting dynamics. One can prove that any real system obtained by a truncation to three modes with a force acting on only one mode has either one stable fixed point or two stable fixed points and one unstable one.

Furthermore the computer shows that for any choice of $\mathbf{f}$ any randomly chosen initial data evolves towards one of the fixed points up to a fairly large value of the "Reynolds number"

$$
r=\sum_{\mathbf{k} \in L}\left|f_{\mathbf{k}}\right| \text {. }
$$

Equations (2.4) have a symmetry property : by changing both the signs of $\gamma_{4}$ and $\gamma_{5}$ they are unchanged (this is the reason why we get two symmatrically placed attractors, see below).

\section{The Stationary Solutions and Their Stability Properties}

The model (2.4) has the following stationary properties:

a) For $0 \leqq r \leqq R_{1}=5 \sqrt{3 / 2}$ there is only one stationary solution $\gamma=\gamma_{0}^{(r)}$ with components

$$
\left\{\begin{array}{l}
\gamma_{1}=\gamma_{2}=\gamma_{4}=\gamma_{5}=0 \\
\gamma_{3}=r / 5
\end{array}\right.
$$

which turns out to be stable and a global attractor for $r$ small enough (this is a particular case of general results on the theory of the Navier-Stokes equations [5]). Numerical evidence suggests that the above solution is a global attractor for all $r \leqq R_{1}$.

b) For $R_{1}<r \leqq R_{2}=\frac{80}{9} \sqrt{3 / 2}$ there are 3 stationary solutions: the old one, $\gamma=\gamma_{0}^{(r)}$, which has become unstable (as a consequence of the crossing of the imaginary axis by one of the eigenvalues of the Liapunov matrix) and two more $\gamma_{ \pm}^{(r)}$, with components

$$
\left\{\begin{array}{l}
\gamma_{1}= \pm \sqrt{\sqrt{6} / 7} \sqrt{r-R_{1}} \\
\gamma_{2}= \pm \sqrt{1 / 7 \sqrt{6}} \sqrt{r-R_{1}} \\
\gamma_{3}=\sqrt{3 / 2} \\
\gamma_{4}=\gamma_{5}=0
\end{array}\right.
$$

which are stable. 

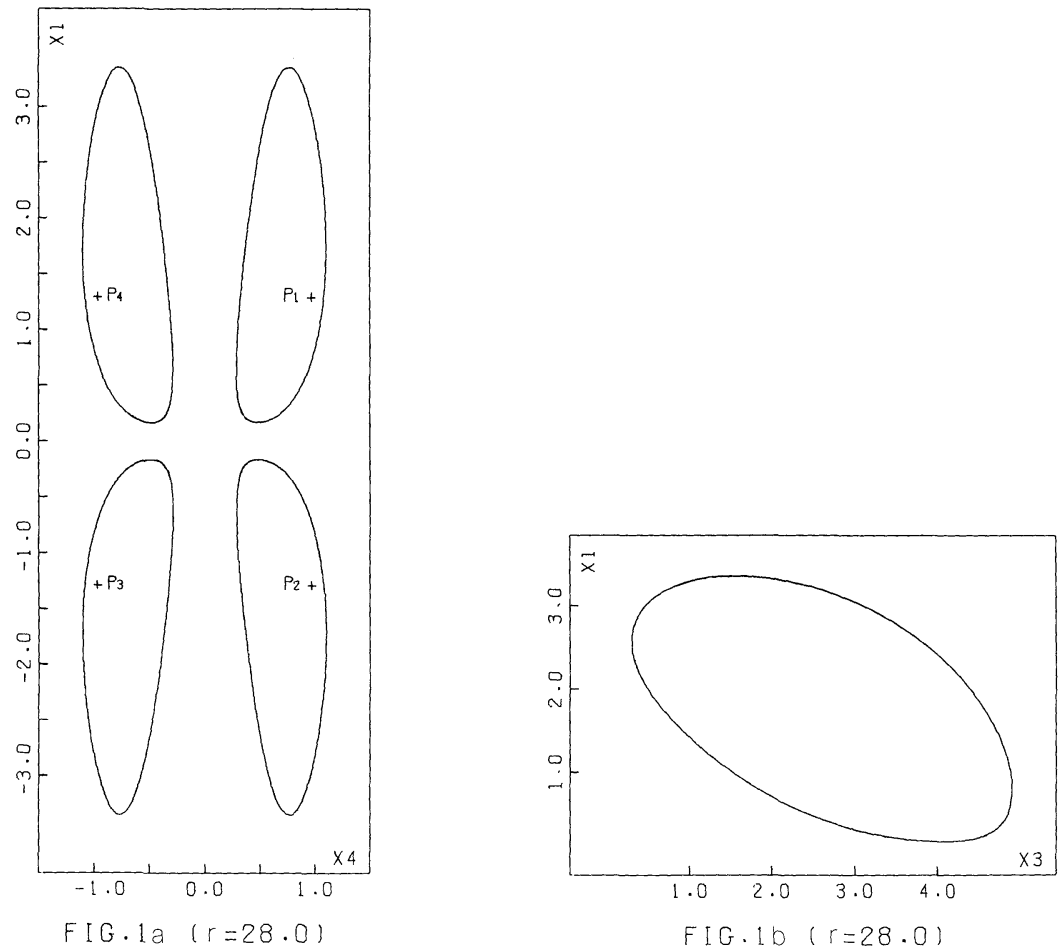

Numerical evidence indicates that any randomly chosen (in the sense of the Lebesgue measure) initial data is attracted by either $\gamma_{+}^{(r)}$ or $\gamma_{-}^{(r)}$ i.e. $\gamma_{ \pm}^{(r)}$ are global attractors.

c) For $r>R_{2}$ there are 7 stationary solutions: the old ones $\gamma_{0}^{(r)}, \gamma_{ \pm}^{(r)}$ and $\gamma_{\varepsilon \sigma}^{(r)}$ with components

$$
\left\{\begin{array}{l}
\gamma_{1}=\varepsilon \sqrt{5 / 3} \\
\gamma_{2}=\varepsilon \frac{3}{80} \sqrt{5 / 3} r \\
\gamma_{3}=\frac{9}{80} r \quad \varepsilon= \pm 1, \quad \sigma= \pm 1 \\
\gamma_{4}=\frac{\varepsilon \sigma}{3} \sqrt{\left(\frac{9}{80} r\right)^{2}-\frac{3}{2}} \\
\gamma_{5}=-\sigma \sqrt{\left(\frac{9}{80}\right)^{2}-\frac{3}{2}} \sqrt{5 / 3} .
\end{array}\right.
$$

The first three are now always unstable, while the new four are stable for $r \leqq R_{3}=22.85370163 \ldots$ and numerically they attract any randomly chosen initial data. 

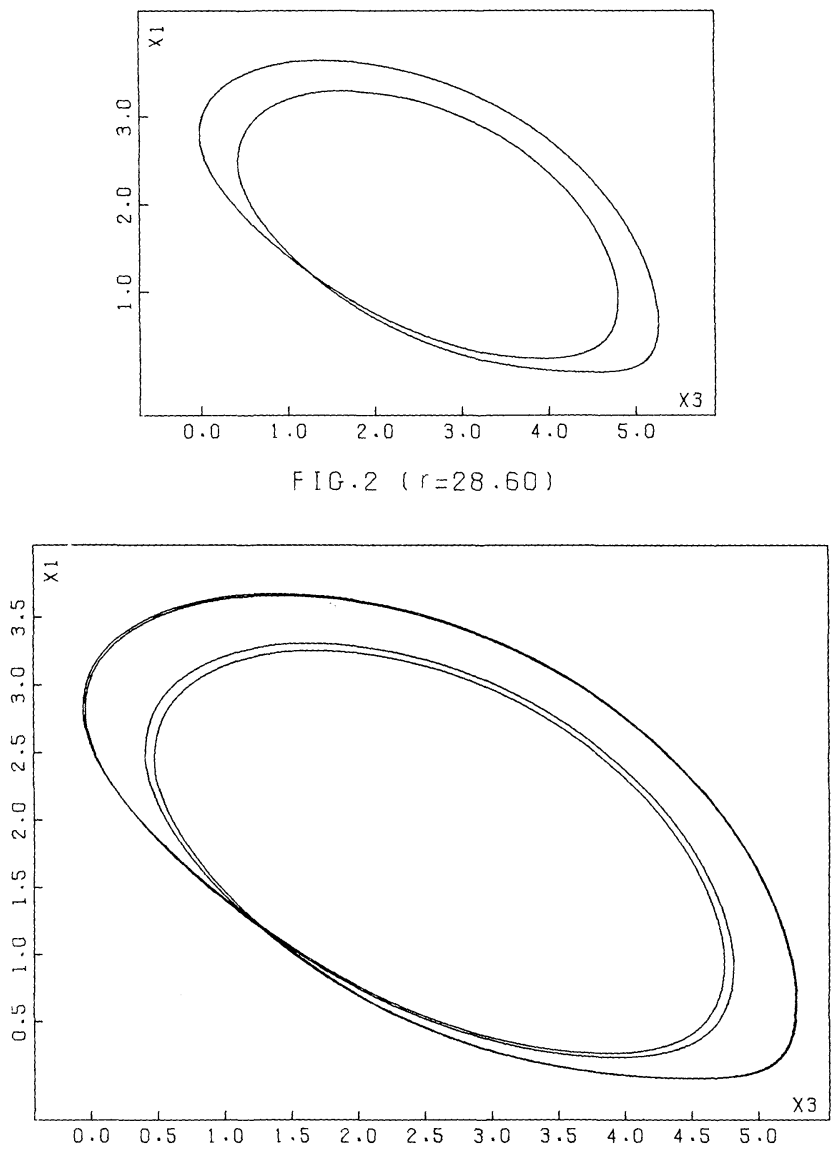

FIG.3 $(r=28.650)$

\section{The Periodic Solutions}

a) At $r=R_{3}$ the four stable stationary solutions become unstable because a pair of complex conjugate eigenvalues crosses the imaginary axis, and four stable periodic orbits around the fixed points $\gamma_{\varepsilon \sigma}^{(r)}$ arise via a Hopf bifurcation (Fig. 1a, b). As predicted by the Hopf theorem the period of such orbits tend to $T=\frac{2 \pi}{\left|\lambda_{0}\right|} \simeq 0.73227$ ( $\lambda_{0}$ being the imaginary part of the eigenvalues which cross the imaginary axis) as $r \rightarrow R_{3}$ from above. These orbits are stable up to $r=R_{4}=28.41 \ldots$ and the computer shows that they attract any point chosen at random.

b) At $r=R_{4}$ the periodic orbits lose stability because an eigenvalue of the Liapunov matrix of the Poincaré map crosses the unit circle in -1 . As predicted by the general theory of bifurcation [6], the computer shows that each one of the orbits considered in a) gives rise to a new periodic orbit with double period (Fig. 2). The new orbits are very similar in shape to the previous ones, only they wind up twice. 


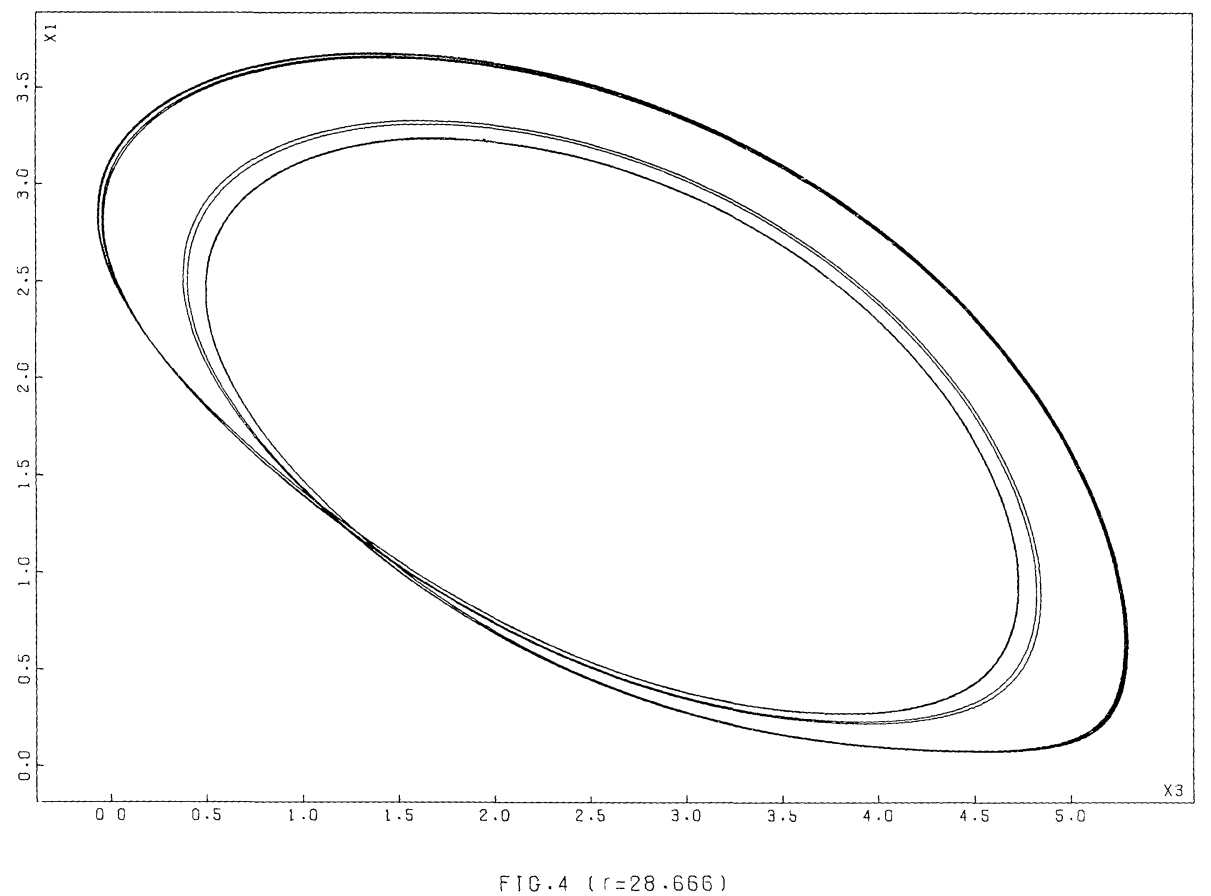

The computer shows two other similar bifurcations, taking place at $r=R_{5}=28.64 \ldots$ and $r=R_{6}=28.66 \ldots$ (Figs. 3,4 ); each time the new orbits wind up around the fixed points twice as many times and the period doubles, up to a period eight times the initial one (see Table 1). We cannot definitely state that there are no other bifurcations of this kind, however the computer shows that up to $r=28.6660$ the orbit arising at $r=R_{6}$ is still there and that

c) in $r=R_{7}, 28.6660<R_{7}<28.6662$, a new kind of orbit appears (Fig. 5). Each of the previous four orbits bifurcates into a new orbit, with period $T^{*} \simeq 3.8$, as shown by Table II. The new orbits wind up around two of the fixed points $\gamma_{\varepsilon \sigma}^{(r)}$, instead of

Table 1. Periods of periodic orbits considered in b), $\S 4$, for different values of $\mathrm{r}$

\begin{tabular}{ll}
\hline$r$ & $T(r)$ \\
\hline 23.00 & 0.73227 \\
26.00 & 0.77458 \\
28.40 & 0.81602 \\
28.42 & 1.63300 \\
28.63 & 1.64512 \\
28.64 & 3.29135 \\
28.660 & 3.29300 \\
28.665 & 6.58686 \\
28.666 & 6.58706 \\
\hline
\end{tabular}

Table 2. Periods of periodic orbits considered in c), $\$ 4$, for different values of $\mathrm{r}$

\begin{tabular}{lr}
\hline$r$ & $T^{*}(r)$ \\
\hline 28.667 & 3.80314 \\
28.690 & 3.80734 \\
28.700 & 7.61811 \\
28.715 & 7.62041 \\
28.716 & 15.24110 \\
28.718 & 15.24208 \\
28.7194 & 30.48554 \\
28.7200 & 60.97203 \\
\hline
\end{tabular}




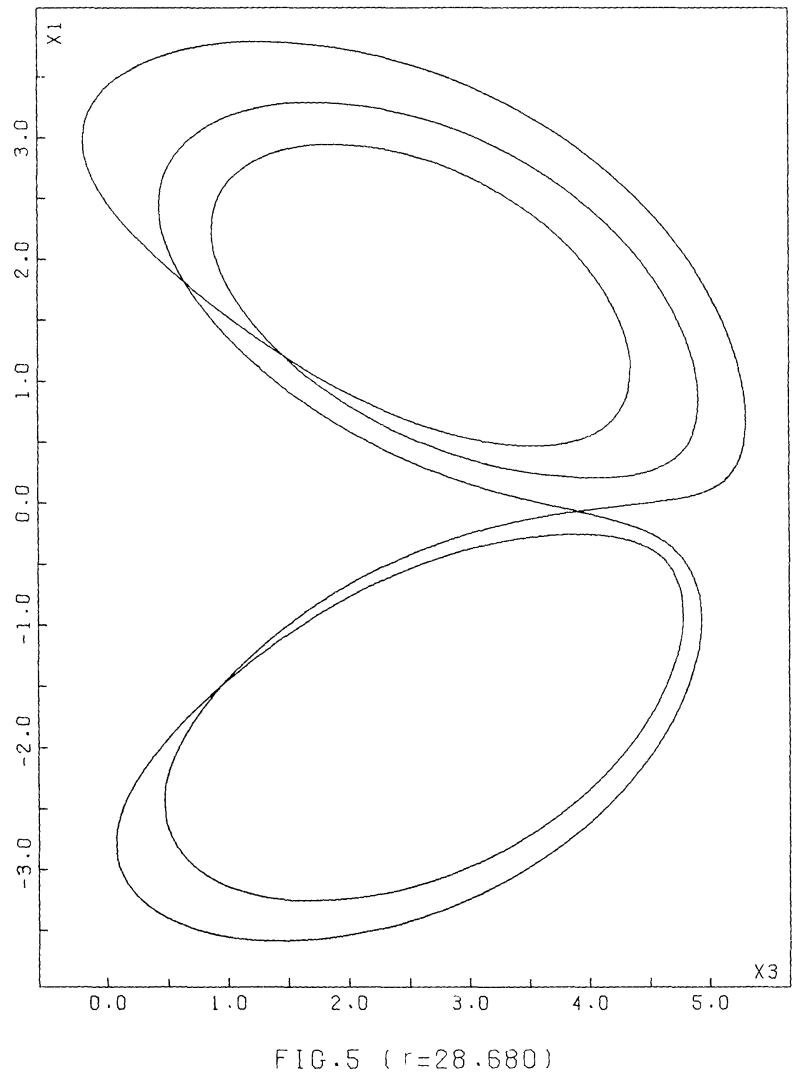

only one like the previous ones. More precisely there are two orbits winding around $\gamma_{++}^{(r)}$ and $\gamma_{-+}^{(r)}$ and the other two around $\gamma_{+-}^{(r)}$ and $\gamma_{--}^{(r)}$ (Fig. 7a $-\mathrm{d}$ ). Increasing $r$ we have a sequence of bifurcations in which each time the period doubles and the number of loops around each fixed point doubles (Fig. 6). We found four further bifurcation points of such nature, for the following values of $r: R_{8} \simeq 28.70, R_{9} \simeq 28.716, R_{10} \simeq 28.719, R_{11} \simeq 28.720$. Since the period doubles each time and the orbits become increasingly intricate requiring higher precision, we did not look for further bifurcations. So we cannot definitely state whether we have just a finite number of bifurcations, or the period keeps doubling and the bifurcation points accumulate. If the latter is the case, and if the bifurcation points accumulate at the rate indicated by the ones we have found, increasing $r$ we would have very soon an orbit with a period so large that it would be impossible to find it on the computer. However we can get some information on how far these bifurcations go in the following way. All the orbits arising in this sequence of bifurcations however complicated, have the following common feature: they wind up around two of the fixed points $\gamma_{\varepsilon \sigma}^{(r)}$, and they make three loops around one point, then two around the other point and so on (Figs. 5-7). So if we consider the graph of, say $\gamma_{1}$, as a function of time, if we wait long enough in order to get the point sit on a periodic orbit, we see a typical bump structure (three positive bumps followed 


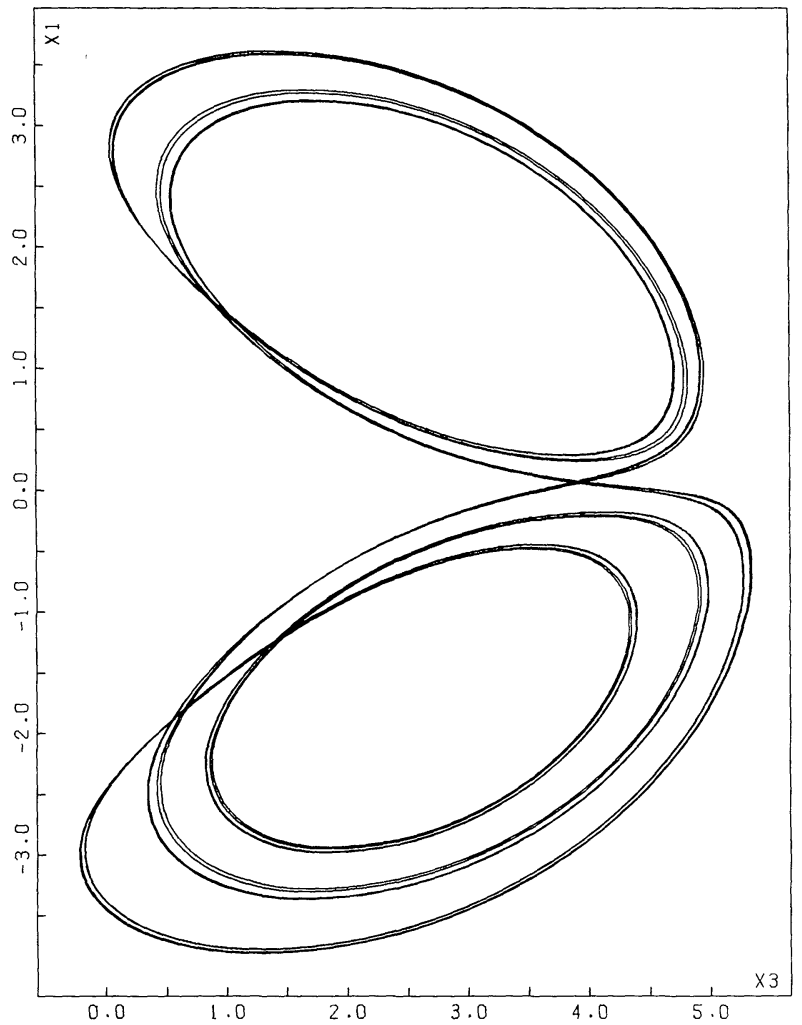

FIG.6 $(r=28.718)$

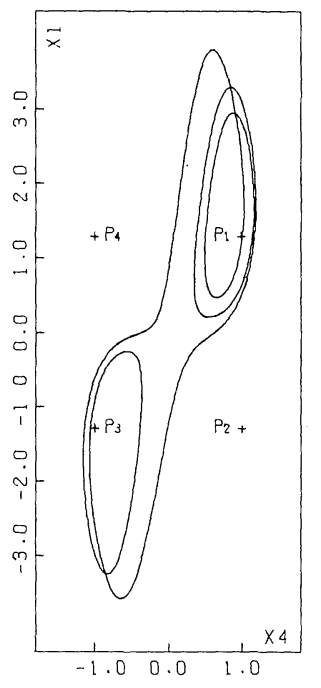

FIG.7a $(r=28.680)$

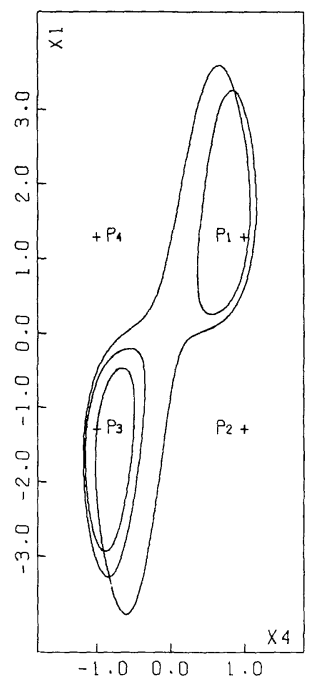

FIG.7b $(r=28.680)$ 


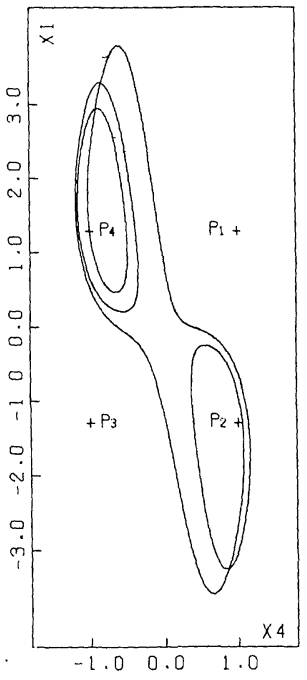

FIG.7C $(r=28.680)$

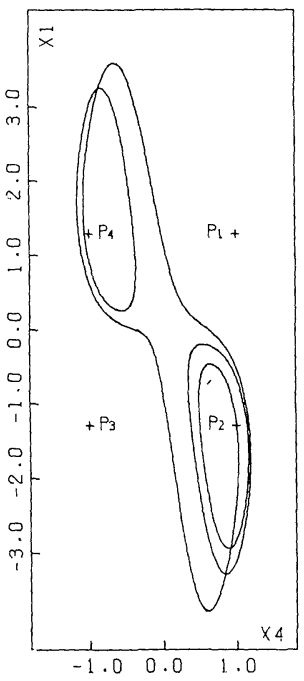

FIG.7d $(r=28.680)$

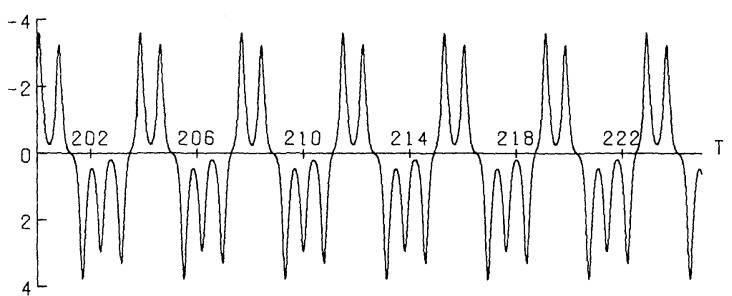

$F[G .8 \mathrm{a} \quad(r=28.70)$

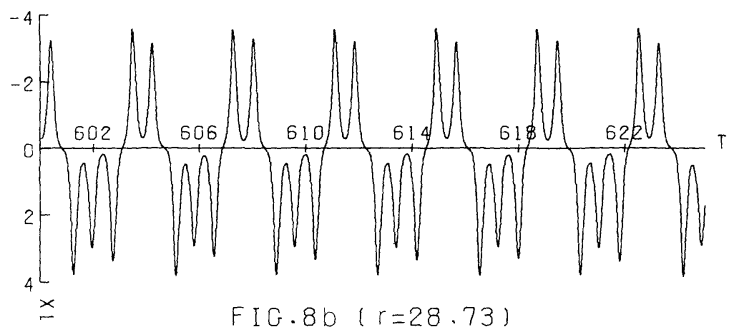

by two negative bumps, for example (Fig. 8a)). We could see that this pattern holds at least up to $r=28.73$ (Fig. 8b).

\section{The Turbulent Regime}

For larger values of the Reynolds number this pattern does not show up any more (for $r=29$ we kept the computer going for 2000 time units), and we observe instead a random behaviour with the system winding up, in the average, the same number of times around the two fixed points. This leads us to conclude that a further bifurcation of a different nature takes place, for $r=R_{12}, 28.73<R_{12}<29$, as a consequence of which the four periodic orbits go over into two attractors, which 


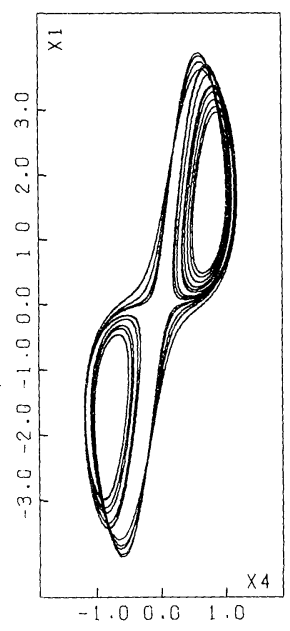

FIG.9 $(r=29.0)$

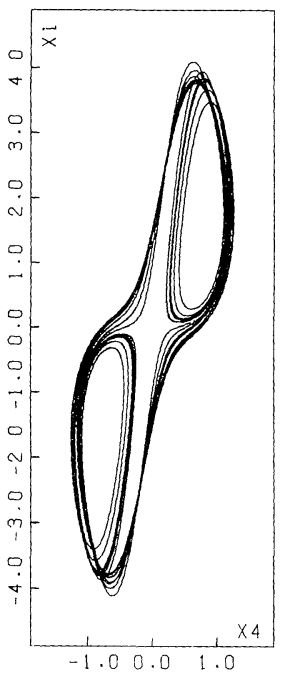

FIG.10a $(r=31.0\}$

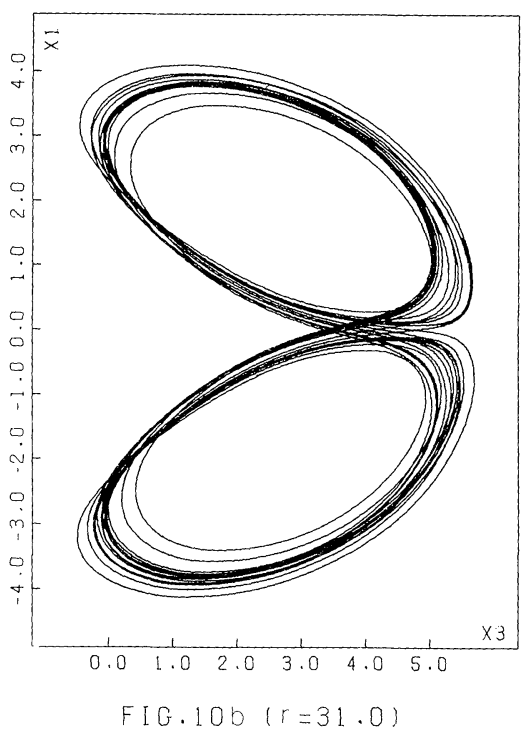

are located respectively in a region surrounding $\gamma_{++}^{(r)}$ and $\gamma_{-+}^{(r)}$, and $\gamma_{+-}^{(r)}$ and $\gamma_{--}^{(r)}$, each attractor being of a shape which resembles very much the union of the two periodic orbits which were in that region before the bifurcation (Figs. 9, 10a, b, 11). This region holds up to $r=R_{13}=33.43 \ldots$ The motion on these attractors seems very random and the pictures of the trajectories resemble very much those found by Lorenz in the analysis of his model [1].

It would be interesting to carry out an analysis like that of Lanford [7] on the Lorenz model to investigate the precise nature of our attractors. Figures 9-11 show projections on different planes of a few trajectories with randomly chosen initial data for different values of the Reynolds number. The behaviour exhibited in the figures looks exactly what one should expect in the neighbourhood of a "strange attractor". 


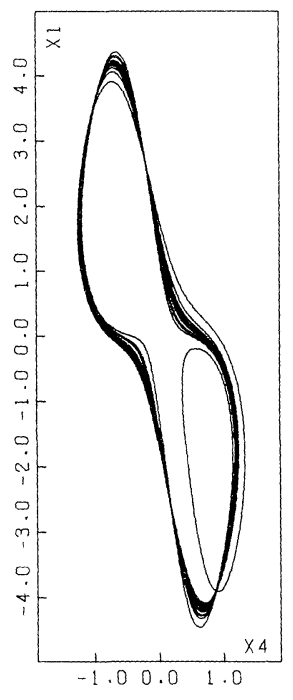

FIG.1) $(r=33.40)$

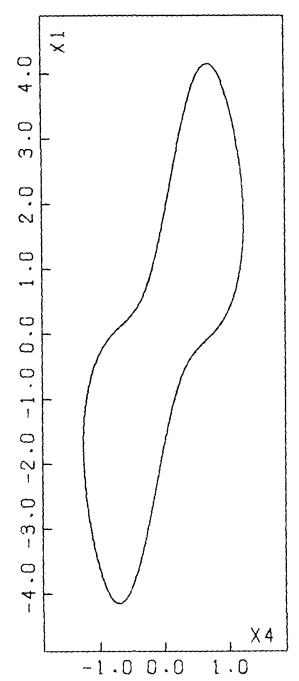

FIG.12a $(r=34.00)$

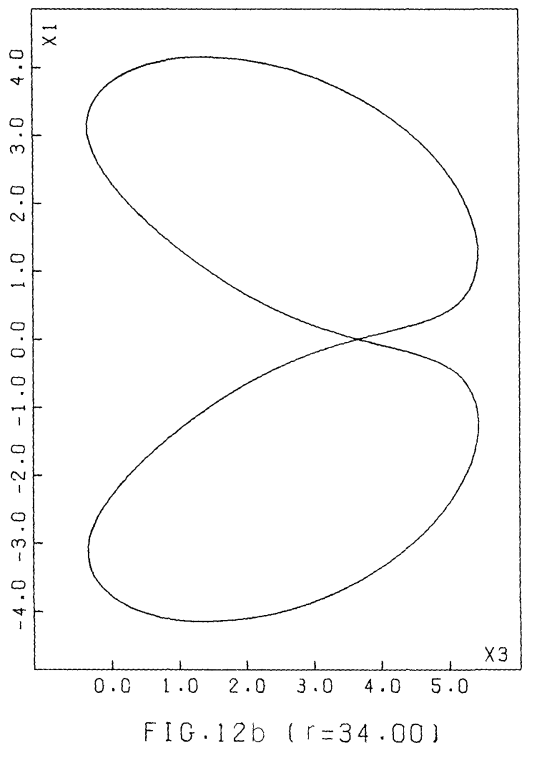

\section{The High $r$ Regime}

It is known [7] that the Lorenz model has, at large values of the Rayleigh number, attractors which look to the computer like simple periodic solutions which behave as global attractors (in the sense, that randomly chosen initial data are attracted by them). Actually, the existence of attracting periodic orbits for large values of the Rayleigh number has recently been shown by Judovich [8]. (The fact that such systems of differential equations possess attractive closed orbits, for small viscosity, seems to be known to engineers, but we do not have the references [9].)

We have also analysed the large $r$ behaviour of our system, and we found that actually for $r>R_{13}$ randomly chosen initial data run eventually into two symmetrically placed simple periodic orbits (which are obtained from each other 
by changing the sign of $\gamma_{4}$ and $\gamma_{5}$ ). Examples of such orbits are shown in Fig. 12a, b.

Figures 10-12 show how the "strange attractor" shrinks to a periodic orbit. The period of these orbits seems to be numerically well defined for $r>R_{13}$ and tends to a finite value $T\left(R_{13}\right) \simeq 1.368$ as $r \rightarrow R_{13}$. Numerical analysis of the eigenvalues of the Poincaré map indicates that the periodic orbit becomes unstable as $r \rightarrow R_{13}$ because one eigenvalue crosses the unit circle at the point +1 . All these facts indicate that we are dealing actually with closed orbits and not with an attractor inclosed in a tiny tube.

The value $R_{13}$ also coincides with the least value of $r$ above which it is possible to close orbits with random initial point so that the period is well defined. For $r>R_{13}$ we are able to measure the period with an accuracy of $10^{-5}$ at least (as well as for the period of the orbits found for $r<R_{12}$ ).

\section{Conclusions}

The most interesting feature of our five modes truncated Navier-Stokes equations is perhaps the way it goes over to "turbulence".

Unlike the Lorenz model it goes through a Hopf bifurcation, and then through a series of bifurcations which lead to increasingly complicated periodic orbits. Stochasticity grows up in some sense "gradually": our analysis of computer data leads us to conclude that we have a transition to a "strange attractor behaviour" through a series of bifurcations which give rise to increasingly complicated attractors made of two very long interwining periodic orbits. If the sequence of bifurcations illustrated at point c), $\S 4$, is actually infinite, after many bifurcations, we would see that these periodic orbits would be very close to being dense on the "strange attractor". The situation which arises in such a way is very interesting and it deserves further analysis.

Acknowledgements. We are deeply indebted to Prof. Giovanni Gallavotti, who began the investigation of the model already in 1975, and gave an unvaluable contribution to our work with his observations and comments.

We also gratefully acknowledge the technical assistance of the staff of Centro di Calcolo, Università di Modena, where the numerical computation were performed.

\section{References}

1. Lorenz, E. N.: J. Athmos. Sci. 20, 130 (1963)

2. Henon, M.: Commun. math. Phys. 50, 69 (1976)

3. Ruelle, D., Takens, F.: Commun. math. Phys. 20, 167 (1971)

4. Ruelle, D.: Lectures at the International School of Mathematical Physics, Camerino (SeptemberOctober 1974)

5. Ladyzhenskaya, O.A.: The mathematical theory of viscous incompressible flows. New York: Gordon and Breach 1969

6. Ioos, G.: Arch. Rat. Mech. Anal. 64, 338 (1977)

7. Lanford, O.E.: Proceedings of Corso C.I.M.E. held at Bressanone (June 1976) (to be published)

8. Judovich, V.I.: (to be published) (in Russian)

9. Gallavotti, G.: Private communication

Communicated by J. L. Lebowitz

Received March 2, 1978 